\title{
Diurnal expression of CONSTANS-like genes is independent of the function of cycling DOF factor (CDF)-like transcriptional repressors in Physcomitrella patens
}

\author{
Tetsuya Ishida', Takumi Sugiyama ${ }^{2}$, Nobumitsu Tabei ${ }^{2}$, Shuichi Yanagisawa ${ }^{1,2, *}$ \\ ${ }^{1}$ Biotechnology Research Center, The University of Tokyo, Bunkyo-ku, Tokyo 113-8657, Japan; ${ }^{2}$ Department of Applied \\ Biological Chemistry, Graduate School of Agricultural and Life Sciences, The University of Tokyo, Bunkyo-ku, \\ Tokyo 113-8657, Japan \\ *E-mail: asyanagi@mail.ecc.u-tokyo.ac.jp Tel: +81-3-5841-3066 Fax: +81-3-5841-8030
}

Received June 16, 2014; accepted August 21, 2014 (Edited by K. Hiratsuka)

\begin{abstract}
The Dof-type transcriptional repressors CYCLING DOF FACTORS (CDFs) directly suppress the expression of CONSTANS (CO), which encodes a key regulator of photoperiodic gene expression, day-length perception and the floral transition in Arabidopsis thaliana. The genes encoding CDF-like (PpDof3 and PpDof4) and CO-like (PpCOL1PpCOL3) proteins are present in the genome of the moss Physcomitrella patens, although $P$. patens lacks the genes encoding homologues of FLAVIN-BINDING KELCH REPEAT F-BOX1 (FKF1) and GIGANTEA (GI), which control the stability of CDFs in A. thaliana. In the current study, we investigated whether the functions of PpDof3 and PpDof 4 are associated with the expression of $P p C O L 1-P p C O L 3$ in $P$. patens. We found that the diurnal expression patterns of PpDof3 and PpDof4 are similar to those of $C D F$ genes and that like CDF1 from A. thaliana, PpDof3 and PpDof4 function as transcriptional repressors. However, targeted disruptions of PpDof3 and PpDof4 did not affect the expression of PpCOL1-PpCOL3, indicating that the expression of COLs is independent of the functions of PpDof3 and PpDof4 in P. patens.
\end{abstract}

Key words: CONSTANS-like gene, cycling DOF factor, Physcomitrella patens, targeted gene disruption.

Dof transcription factors, which possess plant-specific Dof DNA-binding domains, are present in both vascular and non-vascular plants (Moreno-Risueno et al. 2007; Shigyo et al. 2007) and play roles in a variety of biological processes in the plant kingdom (Sugiyama et al. 2012; Yanagisawa 2002). Recently, a Dof transcription factor in Arabidopsis thaliana (Arabidopsis) was identified as a transcriptional repressor of CONSTANS (CO), which encodes a master regulator of photoperiodic gene expression and the floral transition from the vegetative phase to the reproductive phase (Imaizumi and Kay 2006; Samach and Coupland 2000; Song et al. 2010; Valverde 2011); this factor was designated CYCLING DOF FACTOR 1 (CDF1). Subsequently, four Dof proteins in Arabidopsis, CDF2, CDF3, CDF4 and CDF5, were identified as CDF-related factors (Fornara et al. 2009; Imaizumi et al. 2005). All CDFs except CDF4 play redundant roles in repressing $C O$ expression and photoperiodic flowering in Arabidopsis (Fornara et al. 2009). Consistent with the hypothesis that in general, closely related Dof transcription factors share small amino acid motifs outside the highly conserved Dof
DNA-binding domain (Lijavetzky et al. 2003; MorenoRisueno et al. 2007; Shigyo et al. 2007; Yanagisawa 2002), all CDFs except CDF4 have conserved amino acid motifs in their C-terminal regions (Fornara et al. 2009; Imaizumi et al. 2005). Thus, these conserved amino acid motifs are characteristic of CDFs.

Since $C O$ expression is controlled by both light signaling and circadian clocks, and since $\mathrm{CO}$ protein functions as a transcriptional regulator of the expression of FLOWERING LOCUS T (FT), whose product is a mobile signal from the phloem to the meristem, $\mathrm{CO}$ integrates information on day length in leaves and regulates the floral transition in meristems in response to seasonal changes (Samach and Coupland 2000; Imaizumi and Kay 2006; Song et al. 2010; Valverde 2011). CDFmediated transcriptional regulation of the photoperiodic expression of $C O$ as well as post-translational regulation of the stability of CO protein (Jang et al. 2008; Valverde et al. 2004) are key regulatory mechanisms in the COmediated regulatory pathway for photoperiodic gene expression and the floral transition in Arabidopsis (Song et al. 2010; Valverde 2011). CDFs interact

Abbreviations: Arabidopsis, Arabidopsis thaliana; CDF, CYCLING DOF FACTOR; CO, CONSTANS; COL, CONSTANS-LIKE; FKF1, FLAVINBINDING KELCH REPEAT F-BOX1; FT, FLOWERING LOCUS T; GI, GIGANTEA.

This article can be found at http://www.jspcmb.jp/

Published online September 26, 2014 
with FLAVIN BINDING, KELCH REPEAT, F-BOX PROTEIN 1 (FKF1) and GIGANTEA (GI) (Imaizumi et al. 2005; Sawa et al. 2007), which were identified as core components of the CO-mediated regulatory pathway based on the phenotypes of their mutants (Fowler et al. 1999; Imaizumi et al. 2003; Sawa et al. 2007). Furthermore, FKF1, which interacts with GI nuclear protein, controls the stability of CDFs (Imaizumi et al. 2005; Sawa et al. 2007). Thus, CDFs are downstream components of FKF1 and GI in the CO-mediated regulatory pathway in Arabidopsis.

The floral transition is a developmental process unique to flowering plants, while effects of photoperiod on the growth phase transition was found in gymnosperms, ferns and bryophytes as well. In agreement with this fact, CO-like genes (COLs) have been discovered in the moss Physcomitrella patens and the green alga Chlamydomonas reinhardtii as well as flowering plants (Valverde 2011). C. reinhardtii contains the $\mathrm{COL}$ gene $\mathrm{CrCO}$, which plays a pivotal role in photoperiod-regulated control of growth and metabolism in C. reinhardtii and can complement the co mutation in Arabidopsis (Serrano et al. 2009). On the other hand, in the genome of $P$. patens, a model plant studied to gain insights into the evolution of specific gene regulatory networks in plants (Quatrano et al. 2007), three COL genes (PpCOL1-PpCOL3) have been identified (Shimizu et al. 2004; Zobell et al. 2005). PpCOLs are more closely related to Arabidopsis CO-like genes (AtCOL3-AtCOL5) rather than CO itself (Zobell et al. 2005).

We previously reported that two Dof proteins from P. patens, PpDof3 and PpDof4, are probably CDF homologues, because they possess amino acid motifs characteristic of CDFs (Sugiyama et al. 2012). In spite of the presence of the genes encoding COL and CDF-like proteins in $P$. patens, a recent comprehensive analysis of the $P$. patens genome revealed that this moss lacks homologues of FKF1 and GI (Holm et al. 2010). Thus, in the current study, we characterized the roles of PpDof3 and PpDof 4 as transcription factors and investigated the relationship between the functions of PpDof3 and PpDof4 and the expression of PpCOL1-PpCOL3 using gene targeting.

\section{Materials and methods}

\section{Biological materials and growth conditions}

P. patens (Hedw.) Bruch \& Schimp. ssp. patens Tan (Ashton and Cove 1977) was used in this study. Protonema of wild-type and transformed $P$. patens were grown on agar plates containing BCDATG medium $\left(1 \mathrm{mM} \mathrm{MgSO}_{4}, 1.84 \mathrm{mM} \mathrm{KH}_{2} \mathrm{PO}_{4}, 10 \mathrm{mM}\right.$ $\mathrm{KNO}_{3}, 45 \mu \mathrm{M} \mathrm{FeSO}_{4}, 0.22 \mu \mathrm{M} \mathrm{CuSO}_{4}, 10 \mu \mathrm{M} \mathrm{H}_{3} \mathrm{BO}_{3}, 0.23 \mu \mathrm{M}$ $\mathrm{CoCl}_{2}, 0.1 \mu \mathrm{M} \mathrm{Na}_{2} \mathrm{MoO}_{4}, 0.19 \mu \mathrm{M} \mathrm{ZnSO}_{4}, 2 \mu \mathrm{M} \mathrm{MnCl}_{2}, 0.17 \mu \mathrm{M}$ $\mathrm{KI}, 1 \mathrm{mM} \mathrm{CaCl}_{2}, 5 \mathrm{mM}$ ammonium tartrate and $0.5 \%$ glucose) at $25^{\circ} \mathrm{C}$ under a day/night cycle of $16 / 8 \mathrm{~h}$ with approximately
$50 \mu \mathrm{mol}$ photons $\mathrm{m}^{-2} \mathrm{~s}^{-1}$ light. To analyze gene expression, protonema of wild-type and ppdof disruptant lines were regenerated from single protoplasts and grown for 2 weeks on agar plates containing modified BCDATG medium (made with $10 \mathrm{mM} \mathrm{CaCl}$ and $6 \%$ mannitol rather than $1 \mathrm{mM} \mathrm{CaCl}$ and $0.5 \%$ glucose), followed by transfer to agar plates containing BCDATG medium. After 10 days of cultivation on agar plates, protonema samples were sampled every $4 \mathrm{~h}$ for $28 \mathrm{~h}$.

\section{Generation of P. patens disruptants and genomic Southern blot analysis}

To construct gene-targeting vectors, genomic DNA fragments containing PpDof3 or PpDof4 were amplified by PCR using gene-specific primers (Supplemental Table S1) and cloned into pGEM-T (Promega, Madison, WI, USA). Then, the regions encoding the Dof domains of PpDof3 and PpDof4 in the resultant plasmids were replaced with a $35 \mathrm{~S}$ promoterdriven hygromycin resistance gene or a $35 \mathrm{~S}$ promoterdriven kanamycin resistance gene (Sugiyama et al. 2012). Transformation of $P$. patens with these targeting vectors was performed by the polyethylene glycol method, and the transformants were selected on agar plates containing appropriate concentrations of antibiotics according to the method described previously (Sugiyama et al. 2012). Genomic Southern blot analysis was performed with EcoRV-digested genomic DNA from the protonema of the wild type and disruptant lines, as described previously (Sugiyama et al. 2012). PpDof3- and PpDof4-specific DNA probes were prepared by PCR using the gene-specific primers listed in Supplemental Table S1.

\section{Quantitative RT-PCR (qPCR)}

Total RNA was prepared from the protonema samples using a Fruit-mate for RNA Purification kit (Takara Bio, Shiga, Japan) and Trizol reagent (Invitrogen, Carlsbad, CA, USA). Reverse transcription reactions were performed with oligo $(\mathrm{dT})_{15}$ primers or random hexamer primers using SuperScript ${ }^{\circledR}$ II reverse transcriptase (Life Technologies Japan, Tokyo, Japan). Quantitative and semi-quantitative PCR were performed as described previously (Sugiyama et al. 2012). The primers used are listed in Supplemental Table S1.

\section{Protoplasts transient assays}

For transient assays using maize mesophyll protoplasts, a reporter plasmid containing the luciferase reporter gene (LUC), under the control of a LexA-binding site-containing promoter in which eight copies of LexA-binding sites were placed between the $35 \mathrm{~S}$ enhancer and the $35 \mathrm{~S}$ minimal promoter, was utilized (Sugiyama et al. 2012). For construction of effector plasmids, the plant expression vector for LexA-VP16 fusion protein was utilized (Yanagisawa et al. 2003). The DNA sequence encoding the VP16 transcriptional activation domain in the vector was replaced with the entire coding sequence for PpDof3 or PpDof4, which were obtained by PCR using gene-specific primers (Supplemental Table S1). All plasmids 
constructed were verified by DNA sequencing. Co-transfection of reporter and effector plasmids into protoplasts was performed using an internal control plasmid containing uidA [ $\beta$-glucuronidase (GUS) gene] under the control of the maize ubiquitin promoter, as described previously (Yanagisawa 2000). LUC and GUS activity were measured as described previously (Konishi and Yanagisawa 2008).

\section{Results}

\section{Diurnal expression of PpDof3 and PpDof4}

In a previous phylogenetic analysis of the Dof transcription factor family, all CDFs formed a clade together with two other Arabidopsis Dof proteins [the uncharacterized Dof protein AtDof1.3 and COG1, which is involved in phytochrome signaling (Park et al. 2003)]. This clade also contains six Dof proteins from P. patens (PpDof1 to PpDof6) and a Dof protein that is uniquely present in C. reinhardtii (Shigyo et al. 2007). Furthermore, by isolation of full-length cDNAs encoding PpDof1-PpDof6, it was revealed that among Dof proteins from $P$. patens, only PpDof3 and PpDof4 possess conserved amino acid motifs that characterize CDFs (Sugiyama et al. 2012). PpDof3 and PpDof4 do not possess all three motifs characterizing CDFs, but they have two motifs (Figure 1). Thus, PpDof3 and PpDof4 have closest relationships with CDFs. We therefore investigated the expression patterns of $P p D o f 3$ and PpDof4 under long days (LDs) and short days (SDs), together with $P p D o f 1,2,5$ and 6. As shown in Figure 2, PpDof3 and PpDof4 exhibited similar diurnal expression patterns, while $P p D o f 1,2,5$ and 6 did not obviously show any diurnal expression pattern. The expression levels of PpDof3 and PpDof4 were highest at the beginning of the light period and subsequently decreased under both LDs
A

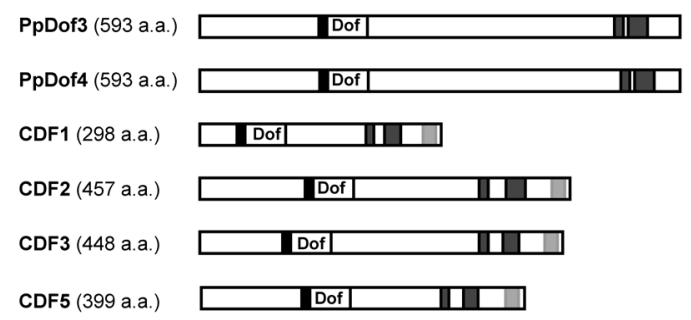

B
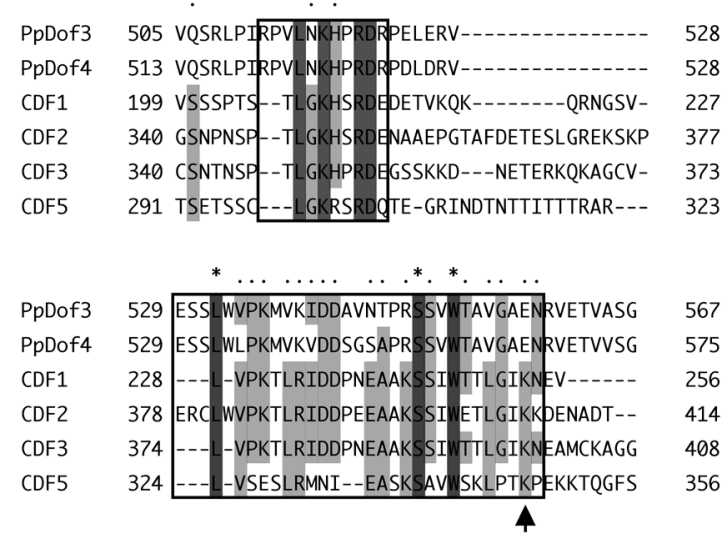

Figure 1. Structures of PpDof3 and PpDof4 from P. patens and CDF1-CDF3 and CDF5 from Arabidopsis. (A) Domain structures. The Dof domain is indicated. A small conserved motif flanking the Dof domain and two motifs conserved in the C-terminal regions of PpDof3, PpDof4, CDF1-CDF3 and CDF5 are also indicated by black and dark grey boxes, respectively. Light grey boxes indicate an additional motif that is conserved in CDFs but not found in PpDof3 or PpDof4. (B) Alignment of the amino acid sequences of the motifs conserved in the C-terminal regions of PpDof3, PpDof4, CDF1-CDF3 and CDF5. Amino acid residues that are completely conserved and highly conserved are indicated in dark and light grey, respectively. The position of $\mathrm{K} 253$, a critical amino acid residue in $\mathrm{CDF} 1$ required for the interaction with FKF1, is indicated by an arrow.
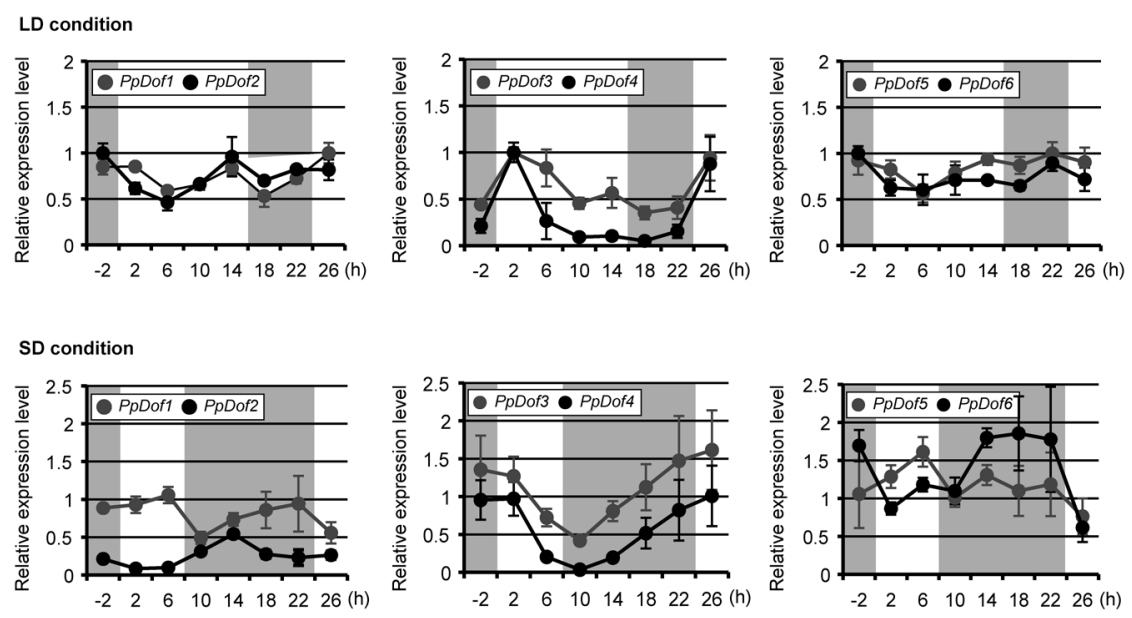

Figure 2. Expression of PpDof1-PpDof6 genes under long (LD) and short day (SD) conditions. RNA was prepared from protonemal colonies sampled every $4 \mathrm{~h}$ for $28 \mathrm{~h}$. Grey shading indicates the night period. Time $(\mathrm{h})$ is expressed as hours after dawn. Values are the mean $\pm \mathrm{SD}$ of three to six biological replicates relative to the levels of $18 \mathrm{~S}$ rRNA. The maximum expression level of each gene under LD conditions was assigned a reference value of 1 unit. 
A

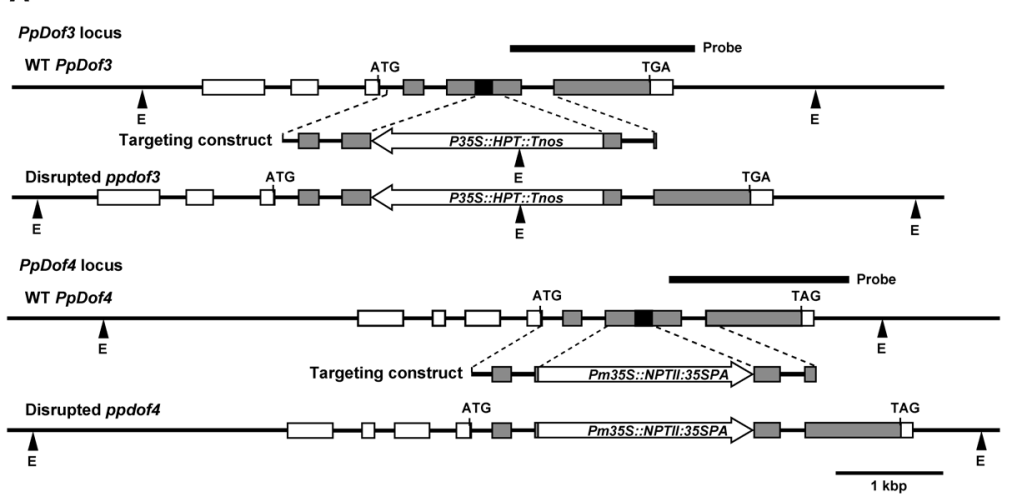

B

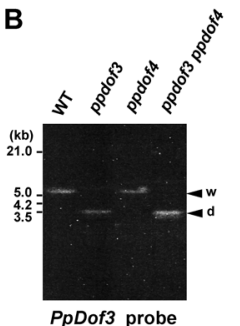

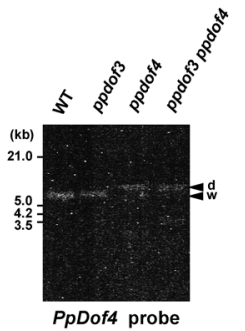

C

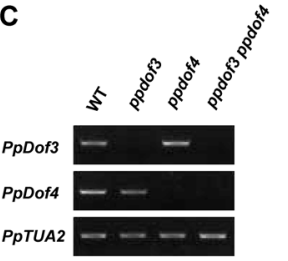

Figure 3. Targeted disruption of $P p D o f 3$ and PpDof4 genes. (A) Schematic representation of the genomic organization of the wild type and disrupted PpDof3 and PpDof4 loci and the gene-targeting vectors. Exons and introns are indicated by boxes and bars, respectively. 5' and 3' untranslated regions, coding regions and the sequence encoding the Dof domain are indicated by white, hatched and black boxes, respectively. Positions of DNA probes used in genomic Southern blot analysis are indicted by red bars. E, EcoRV site; P35S::HPT::Tnos, hygromycin-resistance cassette; Pm35S::NPTII:: Tnos, neomycin-resistance cassette. (B) Genomic Southern blot analysis using EcoRV-digested genomic DNA from the wild-type (WT), single disruptant ( $p p d o f 3$ and $p p d o f 4)$ and double disruptant ( $p p d o f 3$ ppdof4) lines. PpDof3- and PpDof4-specific DNA probes were used. The sizes of DNA fragments originating from the wild type and disrupted $P p D o f 3$ were approximately $6.6 \mathrm{~kb}$ and $3.4 \mathrm{~kb}$, respectively, whereas the sizes of DNA fragments originating from the wild type and disrupted PpDof4 were approximately $7.2 \mathrm{~kb}$ and $8.7 \mathrm{~kb}$, respectively. Positions of DNA fragments from the wild type (w) and disrupted (d) PpDof gene loci are indicated by arrowheads. (C) RT-PCR analysis of PpDof3 and PpDof4 transcripts in the wild-type (WT) and disruptant lines. Transcripts of PpTUA2 (accession number AB096719) were used as a control.

and SDs. This expression pattern is similar to those of $C D F$ genes of flowering plants, including Arabidopsis CDFs (Fornara et al. 2009; Imaizumi et al. 2005), the rice homologue Rdd1 (Iwamoto et al. 2009) and the potato homologues StCDF1.1 and StCDF1.2 (Kloosterman et al. 2013). Although CDF4 and COG1 were also reported to display diurnal expression patterns, their patterns are different from those of CDF1-3 and CDF5 (Fornara et al. 2009). Hence, $P p D o f 3$ and $P p D o f 4$ present characteristics similar to CDFs in terms of mRNA expression pattern as well as protein structure.

\section{Targeted disruptions of PpDof3 and PpDof4 did not affect the expression of PpCOLs}

To investigate the effects of disruption of $P p D o f 3$ and PpDof4 on $P p C O L$ expression, we generated ppdof3 and ppdof4 disruptants by gene targeting (Figure 3 ). We generated single disruptant lines and ppdof3 ppdof4 double disruptant lines using two selectable marker genes (Figure $3 \mathrm{~A}$ ) due to their possibly redundant roles. Southern blot analysis with genomic DNA from these disruptant lines revealed the presence of single bands of the expected sizes (Figure 3B). Furthermore, RTPCR analysis revealed that each gene disruption caused the corresponding intact transcript to be undetectable (Figure 3C). Although we could not find any apparent phenotype of these disruptants, we examined the expression of three $P p C O L$ genes in these disruptant lines. As reported previously (Shimizu et al. 2004; Zobell et al. 2005), $P p C O L 1$ transcript levels increased at dawn and remained high throughout the day but were much lower in the dark in the wild type (WT) under both LDs and SDs (Figure 4). This expression pattern of PpCOL1 was undistinguishable from those of $P p C O L 2$ and $P p C O L 3$. It is worth noting that the expression patterns of the PpCOLs were also similar to that of $\mathrm{CrCO}$ (Serrano et al. 2009) but different from that of CO. In Arabidopsis, CO transcripts primarily accumulate during the dark period under SDs, whereas $C O$ is expressed at moderate to high levels at dawn and dusk under LDs (Yanovsky and Kay 2002). Furthermore, similar to CrCO (Serrano et al. 2009), the absolute levels of $P p C O L$ transcripts were higher under SDs (Figure 4). Importantly, we found that disruption of PpDof3 and PpDof4 did not exert any significant effect on the expression patterns and levels of any COLs under SDs or LDs, indicating that PpDof3 and PpDof4 are not involved in controlling the expression of COLs in P. patens. 

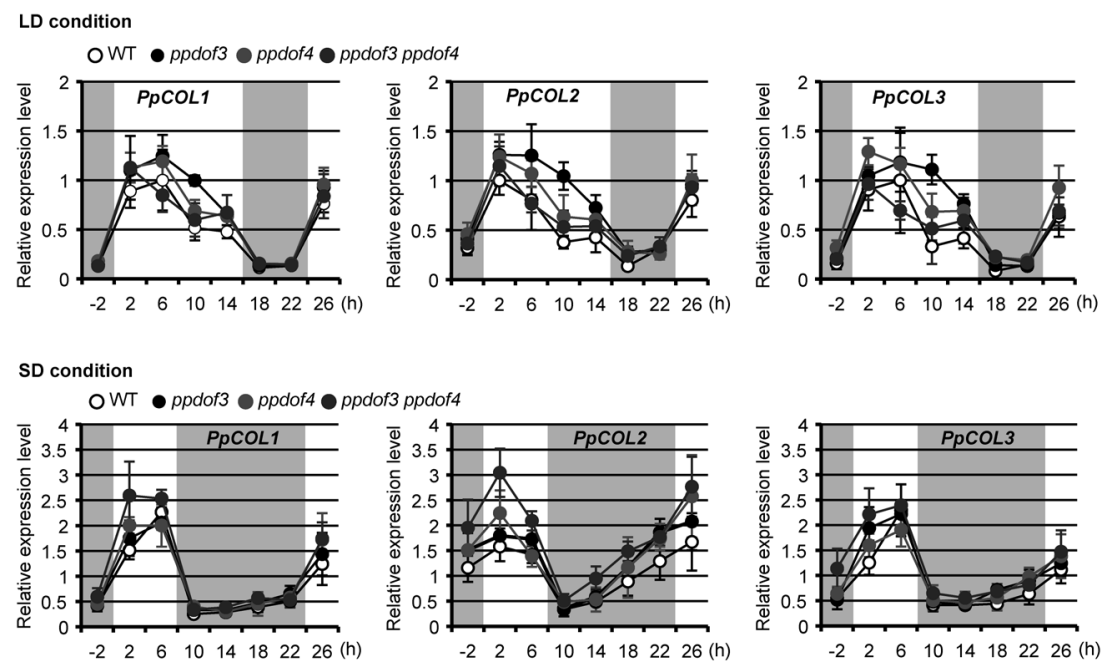

Figure 4. Expression of PpCOL1-PpCOL3 in wild-type (WT) and ppdof mutant $P$. patens under long day (LD) and short day (SD) conditions. RNA was prepared from protonemal colonies sampled every $4 \mathrm{~h}$ for $28 \mathrm{~h}$. Grey shading indicates the night period. Time (h) is expressed as hours from dawn. Values are the mean \pm SD of three to six biological replicates relative to the levels of $18 \mathrm{~S}$ rRNA. The maximum expression level of each gene under LD conditions was assigned a reference value of 1 unit.

A

Reporter

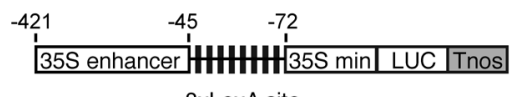

$8 \times$ LexA site

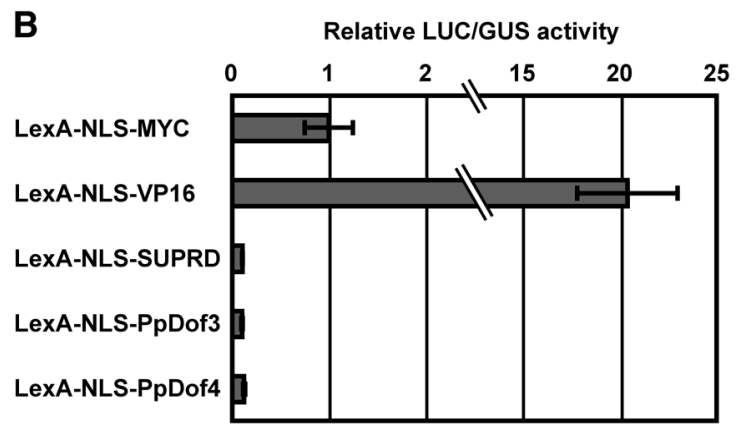

Figure 5. Transcriptional repressor activity. (A) The reporter construct used for the transrepression assay. The reporter construct harbored the LUC gene under the control of eight copies of the LexAbinding site (8xLexA site) located between the $35 \mathrm{~S}$ enhancer and the $35 \mathrm{~S}$ minimal promoter ( $35 \mathrm{~S} \mathrm{~min}$ ). (B) Transrepression assay. The reporter plasmid was co-transfected into maize protoplasts together with an effector plasmid to enable expression of a fusion protein containing the LexA DNA-binding domain, a nuclear localization signal (NLS) and a MYC epitope tag (LexA-NLS-MYC), a VP16 transcriptional activation domain (LexA-NLS-VP16), a repressor domain for SUPERMAN (LexA-NLS-SUPRD), PpDof3 (LexANLS-PpDof3) or PpDof4 (LexA-NLS-PpDof4). An internal control plasmid containing the GUS gene under the control of the maize ubiquitin promoter was also co-transfected into the protoplasts. GUS activity values derived from the internal control plasmid were used to normalize LUC activity. Relative LUC activity is shown as the means $\pm \mathrm{SD}(n=3)$. The relative LUC activity obtained with the effector plasmid for the expression of LexA-MYC protein was set at 1 .

\section{PpDof3 and PpDof4 are transcriptional repressors}

As CDF1 is a transcriptional repressor that directly suppresses CO expression (Imaizumi et al. 2005), we examined whether PpDof3 and PpDof4 also function as transcriptional repressors using protoplast transient assays. When we co-transfected expression vectors for PpDof3 and PpDof4 and reporter plasmids containing the LUC reporter gene under the control of $P p C O L$ promoters, we did not detect any apparent effect of PpDof3 or PpDof4 on LUC activity (Supplemental Figure S1), which is consistent with the fact that disruption of $P p D o f 3$ and PpDof4 did not affect the expression of $P p C O L s$ (Figure 4). Therefore, we next examined the transcription factor activity of PpDof3 and PpDof4 by fusing PpDof3 and PpDof4 to the bacterial DNAbinding protein LexA. We previously succeeded in detecting transcriptional repressor activity of PpDof1 and PpDof2 using this LexA fusion system (Sugiyama et al. 2012). As observed previously (Sugiyama et al. 2012), LexA fused to the VP16 transcriptional activation domain (LexA-VP16) transactivated the LexA-binding site-containing promoter (Figure 5A), while LexA fused to a transcriptional repression domain of the plant transcriptional repressor SUPERMAN (LexA-SUPRD) repressed this promoter. In this assay system, PpDof3 and PpDof4 fused to LexA repressed the expression of $L U C$ under the control of a LexA-binding site-containing promoter (Figure 5). The levels of reduced gene expression induced by LexA-PpDof3 and LexA-PpDof4 were comparable to that induced by LexA-SUPRD. Hence, PpDof3 and PpDof4 are transcriptional repressor like Arabidopsis CDF1 protein.

\section{Discussion}

Our results indicate that like CDFs in flowering plants, the expression of PpDof 3 and PpDof4 is regulated diurnally (Figure 2), and PpDof3 and PpDof4 function as transcriptional repressors (Figure 5). However, targeted 
disruptions of $P p D o f 3$ and PpDof4 did not affect the expression of $P p C O L s$ (Figure 4). Hence, although the diurnal expression of $P p D o f 3$ and $P p D o f 4$, as well as that of $P p C O L s$, implies that all of these genes are involved in the regulation of light signaling- and/or circadian clockassociated processes, it is likely that CDF-like and COlike proteins function independently in $P$. patens.

$\mathrm{CO}$ and COLs are classified into two groups (group $\mathrm{I}$ and group II); group I includes $\mathrm{CO}, \mathrm{CrCO}$ and all PpCOLs as well as AtCOL1-AtCOL5 (Lagercrantz and Axelsson 2000; Valverde 2011). Since overexpression of AtCOL1 and AtCOL2 has little effect on flowering time in Arabidopsis, it was proposed that the roles of CO and COLs do not overlap (Ledger et al. 2001). However, overexpression of AtCOL5 was recently found to affect flowering time (Hassidim et al. 2009). Furthermore, the co mutation in Arabidopsis was complemented by $\mathrm{CrCO}$ and $\mathrm{BvCOL1}$, a sugar beet $\mathrm{COL}$ that is much more similar to AtCOL2 than to CO (Chia et al. 2008; Serrano et al. 2009). Thus, CO and COLs belonging to group I were likely derived from an original gene in the common ancestor of algae, bryophytes and flowering plants, and they appear to be closely related, although $\mathrm{CO}$ might have evolved to play a unique role in the floral transition in flowering plants. Since the expression of COLs is independent of the function of CDF-like Dof proteins in $P$. patens, and since the diurnal expression patterns of the $P p C O L$ s were similar to that of $\mathrm{CrCO}$ but different from that of $\mathrm{CO}$ in Arabidopsis, it is unlikely that CDF-like Dof proteins had played a role in controlling the expression of the original gene of group I $\mathrm{CO} / \mathrm{COL}$ genes in the common ancestor. $\mathrm{CDFs}$ might have been recruited and integrated into the CO-mediated regulatory pathway during evolutionary processes to generate the mechanism underlying the accurate regulation of $C O$ expression. However, at this stage, we could not exclude the possibility that the regulatory role of CDF-like proteins in controlling expression of COLs was lost in P. patens.

Interestingly, the expression levels of the rice $\mathrm{CO}$ homologue Heading date 1 ( $H d 1)$ is not modified in transgenic rice plants in which a transgene for a rice homologue of CDF, rice Dof daily fluctuations 1 (Rdd1), was expressed in sense or antisense orientation (Iwamoto et al. 2009). Thus, it is currently unclear that the CDFmeditated regulation of $\mathrm{CO}$ expression occurs in all flowering plant species. Furthermore, it was very recently reported that $C D F$ homologues from tomato function as transcriptional regulators involved in responses to drought and salt stress as well as flowering-time control in Arabidopsis (Corrales et al. 2014). This unexpected function of tomato CDFs, which is probably independent of the modulation of $\mathrm{CO}$ levels, suggests that CDFs may play another role in flowering plants in addition to the control of flowering. CDF-like Dof proteins may similarly play this additional role in $P$. patens. Identification of the physiological roles of CDF-like Dof proteins in $P$. patens would be necessary to evaluate this speculation. It is noteworthy that in moss, reproductive development begins with the generation of gametophores (Cove 2005), which is promoted by short day lengths (Hohe et al. 2002). However, P. patens lacks an FT homologue (Holm et al. 2010), and the role of PpCOL in reproductive development have not been shown yet.

In flowering plants, CDFs interact with FKF1 and GI (Imaizumi et al. 2005; Kloosterman et al. 2013; Sawa et al. 2007). Thus, we also examined whether PpDof3 and PpDof4 have the potential to interact with Arabidopsis FKF1 and GI using yeast two-hybrid assays (Supplemental Figure S2). The results indicate that unlike CDF1 from Arabidopsis, PpDof3 and PpDof4 did not interact with FKF1 or GI (Supplemental Figure S2). The mutation of a lysine residue within the conserved amino acid motif that characterizes CDFs (the 253th amino acid residue of CDF1) markedly reduces the interaction with FKF1, suggesting that this lysine residue plays a critical role in the interaction with FKF1 (Imaizumi et al. 2005). This lysine residue is not conserved in PpDof3 or PpDof4 (Figure 1b), which is consistent with the lack of an apparent interaction between PpDof3 and PpDof4 and FKF1. The GI-FKF1 system to regulate growthphase transition was very recently found in the liverwort Marchantia polymorpha (Kubota et al. 2014). As P. patens lacks homologues of FKF1 and GI (Holm et al. 2010), identification and characterization of CDF-like Dof proteins and COLs in $M$. polymorpha might provide a new clue to understand the origin of the CO-mediated regulatory pathway.

\section{Acknowledgements}

We thank Dr. M. Hasebe (NIBB, Japan) and Kyowa Hakko Co. (Tokyo, Japan) for generously providing gene targeting vectors and driselase, respectively. This work was supported in part by the Ministry of Education, Culture, Sports, Science and Technology of Japan [Grant-in-Aid for Scientific Research on Innovative Areas no. 21114004] and the Japan Society for the Promotion of Science [grant no. 25252014].

\section{References}

Ashton NW, Cove DJ (1977) The isolation and preliminary characterization of auxotrophic and analogue resistant mutants of the moss, Physcomitrella patens. Mol Gen Genet 154: 87-95

Chia TYP, Muller A, Jung C, Mutasa-Gottgens ES (2008) Sugar beet contains a large CONSTANS-LIKE gene family including a $\mathrm{CO}$ homologue that is independent of the earlybolting (B) gene locus. J Exp Bot 59: 2735-2748

Cove D (2005) The Moss Physcomitrella patens. Annu Rev Genet 39: 339-358

Corrales A-R, Nebauer SG, Carrillo L, Fernández-Nohales P, Marqués J, Renau-Morata B, Granell A, Pollmann S, VicenteCarbajosa J, Molina R-V, et al. (2014) Characterization of tomato 
Cycling Dof Factors reveals conserved and new functions in the control of flowering time and abiotic stress responses. J Exp Bot 65: 995-1012

Fornara F, Panigrahi KCS, Gissot L, Sauerbrunn N, Ruhl M, Jarillo JA, Coupland G (2009) Arabidopsis DOF transcription factors act redundantly to reduce CONSTANS expression and are essential for a photoperiodic flowering response. Dev Cell 17: 75-86

Fowler S, Lee K, Onouchi H, Samach A, Richardson K, Morris B, Coupland G, Putterill J (1999) GIGANTEA: a circadian clock-controlled gene that regulates photoperiodic flowering in Arabidopsis and encodes a protein with several possible membrane-spanning domains. EMBO J 18: 4679-4688

Hassidim M, Harir Y, Yakir E, Kron I, Green RM (2009) Overexpression of CONSTANS-LIKE 5 can induce flowering in shortday grown Arabidopsis. Planta 230: 481-491

Hohe A, Rensing SA, Mildner M, Lang D, Reski R (2002) Day length and temperature strongly influence sexual reproduction and expression of a novel MADS-box gene in the moss Physcomitrella patens. Plant Biol 4: 595-602

Holm K, Källman T, Gyllenstrand N, Hedman H, Lagercrantz U (2010) Does the core circadian clock in the moss Physcomitrella patens (Bryophyta) comprise a single loop? BMC Plant Biol 10: 109

Imaizumi T, Kay SA (2006) Photoperiodic control of flowering: not only by coincidence. Trends Plant Sci 11: 550-558

Imaizumi T, Schultz TF, Harmon FG, Ho LA, Kay SA (2005) FKF1 F-Box protein mediates cyclic degradation of a repressor of CONSTANS in Arabidopsis. Science 309: 293-297

Imaizumi T, Tran HG, Swartz TE, Briggs WR, Kay SA (2003) FKF1 is essential for photoperiodic-specific light signalling in Arabidopsis. Nature 426: 302-306

Iwamoto M, Higo K, Takano M (2009) Circadian clock- and phytochrome-regulated Dof-like gene, $R d d 1$, is associated with grain size in rice. Plant Cell Environ 32: 592-603

Jang S, Marchal V, Panigrahi KCS, Wenkel S, Soppe W, Deng X-W, Valverde F, Coupland G (2008) Arabidopsis COP1 shapes the temporal pattern of $\mathrm{CO}$ accumulation conferring a photoperiodic flowering response. EMBO J 27: 1277-1288

Kloosterman B, Abelenda JA, Gomez MMC, Marian Oortwijn M, de Boer JM, Kowitwanich K, Horvath BM, van Eck HJ, Smaczniak C, Prat S, et al. (2013) Naturally occurring allele diversity allows potato cultivation in northern latitudes. Nature 495: 246-250

Konishi M, Yanagisawa S (2008) Ethylene signaling in Arabidopsis involves feedback regulation via the elaborate control of EBF2 expression by EIN3. Plant J 55: 821-831

Kubota A, Kita S, Ishizaki K, Nishihama R, Yamato KY, Kohchi T (2014) Co-option of a photoperiodic growth-phase transition system during land plant evolution. Nat Commun 5: 3668

Lagercrantz U, Axelsson T (2000) Rapid evolution of the family of CONSTANS LIKE genes in plants. Mol Biol Evol 17: 1499-1507

Ledger S, Strayer C, Ashton F, Kay SA, Putterill J (2001) Analysis of the function of two circadian-regulated CONSTANS-LIKE genes. Plant J 26: 15-22
Lijavetzky D, Carbonero P, Vicente-Carbajosa J (2003) Genomewide comparative phylogenetic analysis of the rice and Arabidopsis Dof gene families. BMC Evol Biol 3: 17

Moreno-Risueno MA, Martinez M, Vicente-Carbajosa J, Carbonero P (2007) The family of DOF transcription factors: from green unicellular algae to vascular plants. Mol Genet Genomics 277: 379-390

Park DH, Lim PO, Kim JS, Cho DS, Hong SH, Nam HG (2003) The Arabidopsis COG1 gene encodes a Dof domain transcription factor and negatively regulates phytochrome signaling. Plant $J$ 34: $161-171$

Quatrano RS, McDaniel SF, Khandelwal A, Perroud P-F, Cove DJ (2007) Physcomitrella patens: mosses enter the genomic age. Curr Opin Plant Biol 10: 182-189

Samach A, Coupland G (2000) Time measurement and the control of flowering in plants. BioEssays 22: 38-47

Sawa M, Nusinow DA, Kay SA, Imaizumi T (2007) FKF1 and GIGANTEA complex formation is required for day-length measurement in Arabidopsis. Science 318: 261-265

Serrano G, Herrera-Palau R, Romero JM, Serrano A, Coupland G, Valverde F (2009) Chlamydomonas CONSTANS and the evolution of plant photoperiodic signaling. Curr Biol 19: 359-368

Shigyo M, Tabei N, Yoneyama T, Yanagisawa S (2007) Evolutionary processes during the formation of the plant-specific Dof transcription factor family. Plant Cell Physiol 48: 179-185

Shimizu M, Ichikawa K, Aoki S (2004) Photoperiod-regulated expression of the PpCOL1 gene encoding a homolog of CO/COL proteins in the moss Physcomitrella patens. Biochem Biophys Res Commun 324: 1296-1301

Song YH, Ito S, Imaizumi T (2010) Similarities in the circadian clock and photoperiodism in plants. Curr Opin Plant Biol 13: 594-603

Sugiyama T, Ishida T, Tabei N, Shigyo M, Konishi M, Yoneyama T, Yanagisawa S (2012) Involvement of PpDof1 transcriptional repressor in the nutrient condition-dependent growth control of protonemal filaments in Physcomitrella patens. J Exp Bot 63: 3185-3197

Yanagisawa S (2000) Dof1 and Dof2 transcription factors are associated with expression of multiple genes involved in carbon metabolism in maize. Plant J 21: 281-288

Yanagisawa S (2002) The Dof family of plant transcription factors. Trends Plant Sci 7: 555-560

Yanagisawa S, Yoo S-D, Sheen J (2003) Differential regulation of EIN3 stability by glucose and ethylene signalling in plants. Nature 425: 521-525

Yanovsky MJ, Kay SA (2002) Molecular basis of seasonal time measurement in Arabidopsis. Nature 419: 308-312

Valverde F (2011) CONSTANS and the evolutionary origin of photoperiodic timing of flowering. J Exp Bot 62: 2453-2463

Valverde F, Mouradov A, Soppe W, Ravenscroft D, Samach A, Coupland G (2004) Photoreceptor regulation of CONSTANS protein in photoperiodic flowering. Science 303: 1003-1006

Zobell O, Coupland G, Reiss B (2005) The family of CONSTANSlike genes in Physcomitrella patens. Plant Biol 7: 266-275 